\title{
VARIABILIDAD EN LA PRÁCTICA CLÍNICA DE LOS CUIDADOS DE LOS SistemAS DE ACCESO VENOSO TOTALMENTE IMPLANTADOS A PARTIR DE LA REVISIÓN DE LOS CUESTIONARIOS Y ENCUESTAS SOBRE SU MANEJO
}

VARIABILITY OF NURSING CLINICAL PRACTICE FOR TOTALLY IMPLANTED VASCULAR ACCESS DEVICES AFTER A BIBLIOGRAPHIC REVIEW OF QUESTIONNAIRES AND SURVEYS ABOUT THEIR MANAGEMENT

\section{Patricia Cerrato Sáez}

Enfermera Emergencias Sanitarias de Castilla y León 


\section{Resumen}

En la actualidad existe una importante variabilidad en los cuidados de los sistemas de acceso venoso totalmente implantados dependientes de múltiples factores.

El objetivo de esta revisión es la descripción de la variabilidad en cuidados de enfermería sobre el manejo de estos dispositivos en los artículos publicados en las bases de datos Web of Science y Scopus que han utilizado cuestionarios y encuestas para analizar dicho cuidado. $Y$ describir los procesos de validación de dichos instrumentos.

Se constata una importante variabilidad en los cuidados de enfermería en los sistemas de acceso venoso totalmente implantados, fundamentalmente en aspectos relativos a las barreras de protección y el lavado y sellado del dispositivo. Sin embargo, se verifica unanimidad en relación al tipo de aguja de acceso. A pesar de ser publicaciones de alto impacto, la mayor parte de los artículos que constituyen la revisión no cumplen la totalidad de items esperables para la validación de sus cuestionarios.

Palabras clave: catéteres de permanencia, dispositivo de acceso vascular, encuestas y cuestionarios.
Abstract

Currently, there is an important variability on totally implanted vascular access devices care that can be explained by diverse factors.

The aim of this review is to describe nursing variability cares by comparing various articles published both in Web of Science and Scopus. In those studies, the authors have used different methods such as questionnaires and surveys so as to analyze and report the aforementioned cares, and also to describe the validation processes of the devices under study.

Thus, variability of nursing clinical practice for totally implanted vascular access devices has been verified, fundamentally, the protection barriers and the washing and blocking techniques. Nevertheless, there is unanimity regarding the kind of needle used to access the device.

Regarding the studies reviewed, despite being high quality publications, most of them did not meet the expected standars for a state-of-the-art survey validation. Particularly due to the insufficient ítems assessed.

Key words: catheters indwelling, vascular access devices, surveys and questionnaires. 


\section{INTRODUCCIÓN}

Un porcentaje elevado de pacientes que requieren atención sanitaria, ya sea en unidades de hospitalización, de urgencias o emergencias y/o de cuidados intensivos, van a necesitar un acceso vascular, ya sea con una finalidad terapéutica o diagnóstica (1).

La Food and Drug Administration (FDA) clasifica los catéteres venosos en $(2,3)$ :

- Catéteres de corta duración. Dentro de este grupo se encuentran los catéteres venosos periféricos (CVP), y el catéter Swan-Ganz, que es un catéter venoso central de corta duración utilizados generalmente para mediciones hemodinámicas $(2,3)$.

- Catéteres de larga duración. Son accesos que dan respuesta a demandas para la administración de tratamientos y nutrición parenteral en enfermos crónicos y pacientes oncológicos. $Y$ se clasifican en:

Catéteres centrales percutáneos parcialmente implantados y tunelizados. El catéter se canaliza por debajo de la piel, pero las aberturas hacia los lúmenes salen a la dermis. El catéter exterior y la piel de alrededor requieren cuidados especiales y debe drenarse periódicamente. Los más frecuentes son el el Hickman en adultos y el Broviac en pacientes pediátricos (4).
Peripherally Inserted Central Catheter (PICC). Son catéteres flexibles que desde un acceso periférico se insertan en la vena Cava Superior $(5,6)$.

Sistemas de acceso venoso totalmente implantados, conocidos coloquialmente como "Ports" o "Reservorios" (2,3). Consta de un reservorio de titanio y una membrana de silicona central, llamada portal o cámara, que puede ser unicameral o bicameral, y permiten hasta 2.000 punciones con una aguja específica de acceso, denominada tipo Huber, cuya característica fundamental es que no posee filo para no dañar la silicona (5). Este dispositivo suele localizarse en la región anterior del pecho (6), pero también se pueden ubicar en la zona anterior de la cresta ilíaca, en la zona femoral (7) e incluso en el brazo introduciendo un catéter totalmente implantado a través de venas periféricas (8). El reservorio está conectado a un catéter que desemboca en la vena cava superior o en la aurícula derecha. El catéter puede ser insertado a nivel profundo, en las venas yugular interna, subclavia y axilar, o superficialmente, en las venas yugular externa, cefálica y basílica (7).

Actualmente las indicaciones para implantar un acceso de estas características en el tratamiento de pacientes oncológicos y/o crónicos son: ausencia o dificultad acusada de acceso venoso pe- 
riférico, necesidad de venopunciones repetidas, manejo de quimioterapia, trasplante de médula ósea, transfusión de sangre y/o hemoderivados, ascitis refractaria, nutrición parenteral, administración de antibióticos de manera prolongada y/o necesidad de analgesia continua ${ }^{(5)}$.

Entre las ventajas de estos dispositivos se distinguen: la posibilidad de ser utilizados durante periodos largos de tiempo, permitiendo mantenerse en funcionamiento durante años, ya sea de manera continua o intermitente (9). Presentan menor número de complicaciones de tipo infeccioso, trombótico y mecánico en comparación con los catéteres centrales percutáneos parcialmente implantados y tunelizados (8,10-12). Otro aspecto de gran consideración es la repercusión de los mismos en la calidad de vida de los usuarios, ya que mejoran la imagen corporal debido a su ubicación subcutánea (13) aportando mayor independencia debido al escaso mantenimiento que requieren y facilitando el desarrollo de una actividad normal (11) lo que genera una alta satisfacción con el catéter implantado $(6,10,14)$.

Entre los principales inconvenientes destacan: para los pacientes, el hecho de que la implantación y retirada del dispositivo requiera intervención quirúr- gica; Y para las instituciones su elevado coste económico (12).

Aunque estos dispositivos son utilizados cada vez con mayor frecuencia por los enfermeros/as $(10,15,16)$ se requiere una serie de cuidados específicos para los cuales es imprescindible estar adecuadamente formado independientemente del contexto profesional en el que se encuentren, ya que su presencia se extiende a otros servicios sanitarios abandonándose su uso exclusivo en servicios de oncología y las unidades de cuidados intensivos $(10,15,16)$.

No obstante, existe una importante controversia en los aspectos relativos a los cuidados de este tipo de catéteres. Cuestiones técnicas como la desinfección de la piel, el lavado y sellado de la luz del catéter, los procedimientos de inserción y retirada de la aguja y la metodología de extracción de muestras, son actuaciones sujetas a una amplia variabilidad dependiente de la unidad asistencial, la institución sanitaria y/o el país de procedencia (13,16-19). Esta marcada diversidad conlleva una escasa estandarización en los cuidados de Enfermería en catéteres totalmente implantados que dificulta el establecimiento de cuidados universales.

El objetivo de este trabajo es identificar los estudios que utilizan instrumentos de medida, ya sean encuestas 
o cuestionarios, para identificar los principales cuidados de Enfermería de los dispositivos de acceso venoso totalmente implantados, y analizar la calidad y el proceso de validación de los mismos.

\section{Metodología}

Se realizó una revisión de la documentación que analiza el conocimiento de los enfermeros/as en el manejo de los dispositivos o sistemas de acceso venoso totalmente implantados en las bases de datos Web of Science y Scopus durante los meses de julio- octubre de 2017.

Para la construcción de la pregunta de investigación se utilizó la estrategia en formato PICO (20): P: población/ pacientes/problema: enfermeros/as res- ponsable de los cuidados de sistemas 0 dispositivos de acceso venoso totalmente implantados. I: intervención/exposición: evaluar el conocimiento técnico en cuidados de los dispositivos. C: comparación: comparar los cuestionarios y encuestas utilizados. O: resultados (outcomes): identificar la calidad y los procesos de validación de los instrumentos. Para ello se utilizaron los Descriptores en Ciencias de la Salud (DeCS): catéteres de permanencia, dispositivos de acceso vascular, encuestas y cuestionarios. $Y$ Medical Subject Headings (MeSH): catheter indwelling, vascular access devices, surveys and questionnaires. Además de otros términos libres combinados con los operadores booleanos: OR y AND mediante la siguiente ecuación de búsqueda (Tabla I).

Tabla 1: Palabras clave y estrategia de búsqueda.

\begin{tabular}{|c|c|c|c|}
\hline Base de datos & Criterios & Estrategia de búsqueda & Resultados \\
\hline Web of Science & $\begin{array}{l}\text { Período de tiempo: } 2007-2017 \\
\text { Dominios de investigación: Science } \\
\text { Technology, Social Science } \\
\text { Todas las bases de datos } \\
\text { Tipos de documento: article, review } \\
\text { Idiomas: english, spanish }\end{array}$ & $\begin{array}{l}\text { ("Questionnaire" [MeSH] OR "survey" [MeSH] } \\
\text { OR "questions" [MeSH] OR "clinical } \\
\text { compatence" [MeSH] OR list OR } \\
\text { "cuestionario" [DeCS] OR "encuesta" [DeCS] } \\
\text { AND "cental venous catheter" [MeSH] OR } \\
\text { "indweling catheter" [MeSH] OR vascular } \\
\text { access port OR totally implanted venous } \\
\text { access device (TIVAD) OR "catéter venoso } \\
\text { central" [DeCS] OR catéter venoso percutáneo } \\
\text { OR catéter venoso subcutáneo) }\end{array}$ & 972 \\
\hline Scopus & $\begin{array}{l}\text { To pubyear } 2007-2017 \\
\text { To subjarea " MEDI" - "NURS - } \\
\text { Limit to languague } \\
\text { "English" OR"Spanish" }\end{array}$ & $\begin{array}{l}\text { ("Questionnaire" [MeSH] OR "survey" [MeSH] } \\
\text { OR "clinical compatence" [MeSH] OR } \\
\text { "cuestionario" [DeCS] OR "encuesta" [DeCS] } \\
\text { AND "cental venous catheter" [MeSH] OR } \\
\text { vascular access port OR totally implanted } \\
\text { venous access device (TIVAD) OR catéter } \\
\text { venoso percutáneo OR catéter venoso } \\
\text { subcutáneo) }\end{array}$ & 526 \\
\hline
\end{tabular}

Fueron considerados susceptibles de inclusión aquellos estudios que incluyeran dentro de la muestra a los en- fermeros/as responsable de los cuidados de catéteres totalmente implantados, Port o Reservorio, que utilizando instru- 
mentos de medida, encuestas, cuestionarios o entrevistas, registrasen los principales cuidados de estos dispositivos, fuesen investigaciones cuantitativas 0 cualitativas y con acceso completo al documento, ya por ser de Open Access o por ser facilitados a través del préstamo interbibliotecario de la biblioteca sanitaria online de Castilla y León, escritos en castellano o inglés.

Se excluyeron los trabajos cuyo objetivo no incluyese los cuidados de los catéteres totalmente implantados, ya por centrarse en otros aspectos como la técnica quirúrgica de implantación o en la satisfacción de los pacientes portadores del dispositivo. También se excluyeron los trabajos de revisión, guías, cartas al editor y/o resúmenes de congresos.

Se realizó un protocolo de revisión sistemática siguiendo la metodología de la declaración PRISMA (Preferred Reporting Items for Systematic reviews and Meta-Analyses) (21).

Para el análisis de la calidad de los estudios cuantitativos se utilizó la lista de ítems de la declaración Strobe (Stregthening the Reporting of OBservational studies in Epidemiology) (22) ya que son estudios descriptivos observacionales transversales que usan el método de encuesta o cuestionario, de manera que se les asignó una puntuación con respecto al cumplimentación de los 22 items de la lista. Los dos estudios cualitativos fueron analizados con la guía CASPe (Critical Appraisal Skills Programme) para estudios cualitativos (23).

Para la evaluación del proceso de validación de los cuestionarios de los estudios cuantitativos se aplicaron los criterios de viabilidad, fiabilidad, validez y sensibilidad al cambio (24). Y para los estudios cualitativos la determinación por parte de los autores sobre el cumplimiento de las recomendaciones COREQ (Consolidated criteria for Reporting Qualitative reseach) (25).

\section{REsultados}

\section{Proceso de selección}

A partir de la búsqueda bibliográfica en las fuentes de datos se obtuvieron 1498 documentos (972 procedentes de WOS y 526 procedentes de Scopus). Añadimos a estos resultados 21 documentos procedentes de otras fuentes como DOAJ y Cuiden, y repositorios de las Universidades de Valladolid, Salamanca y León: Uvadoc, Gredos y Buleria respectivamente (literatura gris). A continuación se descartaron 342 documentos identificados como duplicados a través del gestor bibliográfico Mendeley. Tras la lectura de título y abstract se eliminaron 1011 documentos. Seguidamente los documentos fueron sometidos a los criterios de selección, retirándose 126 artícu- 
los por no estar relacionados con el objetivo de la revisión, 21 documentos por no obtenerse a texto completo y 7 estudios por estar en idioma diferente al inglés y el castellano. De esta manera se clasifi- caron 12 artículos para la realización de la revisión, donde 10 son artículos de investigaciones cuantitativas y 2 son investigaciones cualitativas.

Figura 1: Diagrama de flujo de la metodología de búsqueda.

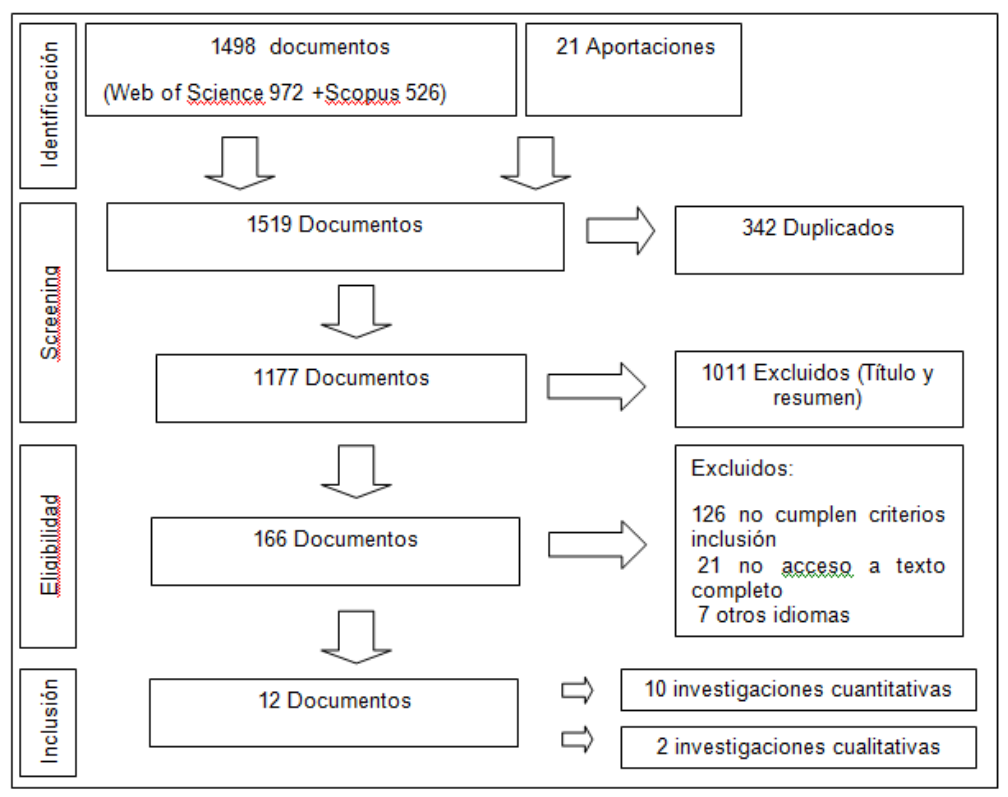

\section{Principales cuidados}

En los estudios revisados los catéteres totalmente implantados son en su gran mayoría de una cámara y una sola luz, generalmente localizados en el tórax y preferiblemente insertados en la vena subclavia $76 \%$ (17) seguido de la yugular interna $44,1 \%$ (26) y localizados mayoritariamente en el lado derecho (27). Con el objetivo principal de ser utilizados para la administración de quimioterapia, aunque este acceso también puede ser utilizado posteriormente para la extracción de muestras y la administración de otros tratamientos como fármacos, fluidos, transfusiones sanguíneas y nutrición pa- renteral $(17,26,28,29)$. No obstante, hay profesionales en contacto con los dispositivos que desconocen su ubicación anatómica (30), y profesionales, como los de sala de Urgencias y Medicina Interna, que ignoran la posibilidad de administrar otras soluciones que no sea quimioterapia (18).

Algunos autores recomiendan el uso de anestésico local con el fin de disminuir el dolor que pueda provocar el pinchazo de acceso, sin embargo en los estudios analizados se describen tasa muy bajas, $10-20 \%$, de desarrollo de esta técnica $(16,17,30,31)$. 
Antes de proceder al acceso del dispositivo los profesionales han de adoptar las barreras de protección adecuadas. En primer lugar, se debe de proceder al lavado de manos con jabón o alcohol antiséptico, esta primera barrera de protección no se desempeña en el $100 \%$ de los accesos al dispositivo $(16,19,28)$. A continuación se debe de desinfectar la piel del paciente por encima de la cámara del reservorio antes de acceder al mismo. Las opciones de desinfección utilizadas con mayor frecuencia son povidona yodada seguida de clorhexidina $(16,17,19,26)$ excepto en los estudios de Broadhurst donde se registra una tasa de uso de chorhexidina del $94,7 \%$ (32) y el estudio de Simon que encabeza la lista el propanol con una tasa de $24 \%$ como antiséptico de elección (29). Destaca el uso de alcohol de $70^{\circ}$ por los profesionales de Urgencias y Medicina Interna como solución antiséptica utilizada habitualmente (18). Una vez aplicadas cualquiera de las soluciones antisépticas se debe esperar hasta que se seque la solución antes de la inserción de la aguja. Este aspecto solo es registrado en los cuestionarios de Fernández de Maya y Broadhurst, donde sólo el 4,9\% espera el tiempo suficiente cuando se usa povidona yodada (cuyo tiempo de espera recomendado son $2-3$ minutos) y el $83,9 \%$ cuando se usa clorhexidina (cuyo tiempo de espera es de 30 segundos). Esta práctica inadecuada se relaciona con un riesgo aumentado de dermatitis $(16,19,32)$.

Siguiendo con las medidas de protección y antes de proceder a la punción de la cámara es recomendable el uso de guantes. Se observa una amplia variabilidad en el tipo de guantes usados, pues en el estudio de Simon desarrollado en Alemania, Austria y Suiza un $38 \%$ de los profesionales constatan el uso de guantes estériles, mientras que en el estudio italiano es un $56 \%$ y en los estudios españoles se registra una tasa superior al $95 \%(16,19,29)$.

El acceso al dispositivo se debe de realizar con una aguja específica denominada tipo Huber, que se inserta perpendicularmente al reservorio (18). Se observa un uso generalizado de este tipo de aguja por los profesionales en contacto habitual con los dispositivos. Sin embargo, los profesionales que tienen un contacto más eventual, manifiestan que en ocasiones necesitan confirmar con otros compañeros el tipo de aguja de acceso y la técnica de inserción (31). Llama la atención los resultados de los estudios brasileño y turco con un $21,4 \%$ y un $44,4 \%$ respectivamente de profesionales que desconocen el tipo especial de aguja de acceso (18,30).

Para evitar el desplazamiento de la aguja insertada se utilizan apósitos 
para su inmovilización. En el $80-90 \%$ de los casos se utiliza gasas estériles (1619,26,29,32) fijadas con diferentes tipos de apósitos, ya sean transparentes, quirúrgicos, esparadrapo adhesivo etc ${ }^{(16,19)}$.

Una vez acabada la infusión de las soluciones requeridas, se procede a los cuidados de lavado y sellado del catéter antes de retirar la aguja Huber. En relación al procedimiento de lavado y sellado se registra una amplia variabilidad. Se recomienda no usar jeringas de tamaño menor de $10 \mathrm{ml}$, ya que podrían generar una presión tan intensa que provocase la desconexión del portal o cámara del catéter (30). En el $90 \%$ de los casos los profesionales aseguran seguir esta recomendación $(1,16,17)$. Las soluciones utilizadas para el lavado y sellado en los estudios analizados son:

- Solución salina fisiológica 0,9\% seguida de la administración de heparina en un 55-72\% de las ocasiones $(16,19,29)$.

- Solución salina fisiológica 0,9\% seguida de solución heparinizada en un $61,1 \%$ en el estudio de Kapucu (28).

- Solución salina fisiológica en un $21 \%$ de los casos según el estudio de Keogh y Ullman $(1,26)$.

- Solución heparinizada en aproximadamente el $20 \%$ de los casos en los estudios de Ullman y $\operatorname{Simon}(26,29)$.

Durante el proceso de sellado destacan la técnica de sellado con "presión positiva" que se realiza en el $92 \%$ de los centros italianos analizados en el estudio de Dal Molin (17), mientras que en los estudios de Fernández de Maya la técnica de "push-pull" (infundir- pararinfundir) se realiza habitualmente un $12,3 \%$ de las ocasiones $(16,19)$.

Este tipo de catéter además de facilitar la infusión de soluciones, también permite la extracción de muestras. Este procedimiento se llevaría a cabo antes de la infusión de soluciones desechando previamente una cantidad de sangre antes de la toma de la muestra. En el estudio de Fernández de Maya un $34,1 \%$ de los centros descartan $10 \mathrm{ml}$ de volumen y un $31,2 \%$ desecha $5 \mathrm{ml}$ de sangre (16).

Los profesionales en enfermería al cuidado del pacientes portadores de sistemas de acceso totalmente implantados han de saber identificar las complicaciones relacionadas con los mismos (27), así como conocer su manejo. En los estudios revisados las complicaciones mencionadas son:

Alteración en la integridad de la piel. Ante esta complicación se procede al cambio de antiséptico para usar el menos lesivo (32). 
Oclusión de la luz del catéter (26).

Para conseguir su desobstrucción se utiliza fármacos trombolíticos, urokinasa 0 streptokinasa, y anticoagulantes, heparina (16).

Fiebre. En el $90 \%$ de los centros que participan en el estudio de Simon esta protocolizados la extracción de muestra sanguínea ante la hipertermia (29).

Según los estudios de Pires, Kapucu y Özden existe una diferencia significativa en el nivel de conocimientos y cuidados de los dispositivos en función de si los profesionales han recibido o no formación y entrenamiento en la materia $(18,28,30)$ influyendo también la frecuencia de esta actualización según Broadhurst (32).

\section{Evaluación de los instrumentos} utilizados en los estudios

Los estudios seleccionados para la revisión se valieron de encuestas o cuestionarios ad hoc elaborados para medir el nivel de conocimiento de los enfermeros/as en cuidados de los dispositivos de acceso venoso totalmente implantados en sus diferentes ámbitos de desarrollo.

Tras la aplicación de la lista de verificación Strobe a los estudios que constituyen la revisión, se observó que el documento más antiguo: Dal Molín de 2009 es el que menos ítems cumple,
11/22, mientras que el artículo de Keogh del año 2015 es el de mejor valoración con 22 items sobre 22 (Anexo I). En ambos artículos cualitativos, se observó carencia en lo relativo a la reflexividad según la guía CASPe (Anexo II).

Dentro de los trabajos de investigación cuantitativa, en las aportaciones de Dal Molin (17) no se mencionaba técnica alguna de validación del instrumento de medida utilizado. En el trabajo de Russell y col. (27) se especificaba que se realizaron modificaciones a partir de una encuesta diseñada por otro autor, y que después fue sometida a un pilotaje por dos enfermeras expertas en prácticas relacionadas con oncología. En el estudio de Keogh (1) a partir de un instrumento estadounidense se realizaron adaptaciones para aplicarlo al objetivo y la población del estudio, sólo se especificaba la realización de pruebas de validez de la lógica y del contenido a través de los expertos y el pilotaje en una muestra, pero no se mencionaban otras estrategias. En el estudio de Özden \& Çaliskan (30) se especificaba que no se llevaron a cabo pruebas de validez ni de viabilidad, aunque sí se pilotó el test previamente con 5 enfermeras del Hospital de Día Oncológico que permitió evaluar las preguntas incomprensibles, poco claras y disfuncionales. En el estudio de Simon (29) tanto el estudio como la encuesta fueron 
evaluados y aprobados por la German

Society of Paediatric Oncology and Hematology (GPOH). Los dos artículos de Fernández de Maya $(16,19)$ se valieron de la misma herramienta, que fue pilotada en dos hospitales para evaluar la lógica, la validez del contenido y su aplicabilidad. En el artículo de Broadhurst (32) se realizó la validación del contenido, mediante el Content Validity Index (CVI) Ilevado a cabo por 7 expertos de USA, Canadá y Australia, revisando las preguntas del cuestionario, realizándose también un test de viabilidad por 8 profesionales clínicos procedentes de los mismos países mencionados anteriormente. En el estudio de Kapucu (28) se determinó el Internal Consistency Coefficient con un valor de 0,654 , y el cuestionario fue sometido a la opinión de un equipo de expertos constituido por 2 enfermeras de oncología y 5 académicos, llevándose a cabo una encuesta preliminar a 5 enfermeras evaluando la penetrabilidad cognitiva y la conveniencia de la herramienta. El último artículo de corte cuantitativo está representado por el trabajo desarrollado por Ullman (26) donde se aplicó la Scale Content Validity Index (S-CVI) por 5 expertos en la materia para la validación del contenido, y para evaluar su viabilidad y fiabilidad se pilotó la herramienta en un hospital (Anexo III).
En el estudio cualitativo de N. Pires y I. Vasques (18) la pregunta inicial de la que emergieron los distintos aspectos relacionados con el manejo del catéter no fue sometida a evaluación. Sin embargo, en el estudio de Filbet (31) se pilotó la calidad del cuestionario, evaluando la comprensión y precisión de las preguntas a través de dos entrevistas preliminares y especificando la cumplimentación de las recomendaciones COREQ (25) (Anexo IV).

\section{Discusión}

Discusión sobre los principales cuidados

Según la bibliografía revisada los catéteres totalmente implantados suelen localizarse en la región del tórax en el lado derecho. Suelen implantarse para la administración de quimioterapia, pero una vez implantado también son usados para la obtención de muestras y la administración de soluciones y/o fármacos $(17,26-29)$.

En los estudios analizados apenas se contempla el uso de anestésico local previo al acceso del catéter $(16,17,30,31)$. Una futura línea de investigación sería la indagación de las razones de esta situación. 
A pesar de las múltiples campañas por parte de la OMS sobre la importancia, las indicaciones y la técnica del lavado de manos (33), en algunos de los estudios analizados se observa que este procedimiento de higiene básica no fue aplicado en todos los accesos a dispositivos (16,19,28). Es imprescindible que los profesionales sanitarios adopten el lavado de manos sistemático como primera barrera de protección ante cualquier tipo de intervención (34).

En la actualidad, la clorhexidina es considerada solución antiséptica de elección por su potente acción bactericida y fungicida con efecto inmediato (15-30 segundos) y con un efecto residual de hasta 6 horas, con escasas reacciones alérgicas descritas y con una buena relación coste-efectividad (35,36). A los autores les sorprende que en los artículos revisados no sea utilizada de manera extendida y generalizada.

En los estudios analizados se observa una importante variabilidad en el procedimiento de lavado y sellado del catéter (1,16,19,26-29). Entendemos que esta práctica está sujeta a los protocolos específicos de cada institución. No obstante, se considera que es conveniente el uso de solución salina fisiológica para el lavado y alguna solución anticoagulante en el sellado para evitar la formación de trombos (37).
Los profesionales sanitarios estamos en constante actualización con el objetivo de garantizar la prestación de cuidados en salud de calidad (38), y en este caso en particular relativo a los catéteres totalmente implantados, es fundamental la formación continua tal como reflejan los estudios de Özdan, Broadhurst, Pires y Kapucu (18,28,30,32).

Discusión sobre la evaluación de los instrumentos utilizados en los estudios

A pesar de que todos los artículos que constituyen la revisión son publicaciones con JCR entre Q1 y Q4, todos ellos utilizan cuestionarios ad hoc, donde solo los estudios de Ullman y Kapucu $(26,28)$ cumplen tres de los cuatro criterios de validación de un cuestionario: viabilidad, fiabilidad, validez y sensibilidad al cambio (24,39). El trabajo cualitativo de Filbet (31) es el único en el que se cumplen las recomendaciones COREQ. Desde el ámbito académico se hace hincapié en la importancia de las publicaciones de alto impacto y el uso de cuestionarios validados. En este caso, comprobamos que los artículos que constituyen esta revisión, a pesar de tener un alto factor de impacto no todos cumplen con las expectativas académicas. 


\section{BiBLIOGRAFÍA}

1. Keogh S, Flynn J, Marsh N, Higgins N, Davies $\mathrm{K}$, Rickard $\mathrm{CM}$. Nursing and midwifery practice for maintenance of vascular access device patency. A cross-sectional survey. Int J Nurs Stud [Internet]. 2015;52(11):1678-85. Available from: http://dx.doi.org/10.1016/j.jnurstu.2015.07.001

2. Wu O, Boyd K, Paul J, McCartney E, Ritchie M, Mellon D, et al. Hickman catheter and implantable port devices for the delivery of chemotherapy: a phase II randomised controlled trial and economic evaluation. $\mathrm{Br} \mathrm{J}$ Cancer [Internet]. 2016;114(9):979-85. Available from: http://www.nature.com/doifinder/10.1038/bjc.2016.76

3. Seisdedos Elcuaz R, Conde García M. C, Castellanos Monedero JJ, García-Manzanares Vázquez-de Agredos A, Valenzuela Gámez JC, Fraga Fuentes M. D. Infecciones relacionadas con el catéter venoso central en pacientes con nutrición parenteral total. Nutr Hosp [Internet]. 2012 [cited 2017 Oct 3];27(3):775-80. Available from: ht tp://scielo.isciii.es/scielo.php? pid=S0212-16112012000300014\&script=sci_arttext\&tlng=pt

4. Gow KW, Tapper D, Hickman RO. Between the lines: The 50th anniversary of long-term central venous catheters. Am J Surg [Internet]. 2017;213(5):837-48. Available from: http://dx.doi.org/10.1016/j.amjsurg.2017.03.021

5. Morán Cabal P. Reservorios de acceso venoso central en pacientes pediátricos oncológicos. Experiencia y complicaciones. [Internet]. 2016. Available from: http://156.35.2.127/dspace/ handle/10651/37610

6. Samad AMA, Ibrahim YA. Complications of Port A Cath implantation: A single institution experience. Egypt J Radiol Nucl Med. 2015;46(4):90711.

7. Imagawa Fonseca I, Krutman $M$, Nishinari K, Yazbek G, Passos Teivelis M, Zottele Bomfim G, et al. Brachial insertion of fully implantable venous catheters for chemotherapy: complications and quality of life assessment in 35 patients. Einstein (São Paulo) [Internet]. 2016;14(4). Available from: h t t p: / / ww w. scielo.br/scielo.php? pid $=S 1679-45082016000400473 \&$ script $=$ sci_arttext\&tlng=pt

8. Shiono M, Takahashi S, Takahashi M, Yamaguchi T, Ishioka C. Current situation regarding central venous port implantation procedures and complications: a questionnaire-based survey of 11,693 implantations in Japan. Int J Clin Oncol. 2016;21(6):1172-82.

9. Corrella Calatayud J, Fuster Diana C, Vázquez Prado A, Corella Mas J, Galbis Caravaja $J$, Rabadén Hontangas R. Reservorios, Acceso Venoso De Larga Duración. Abordaje Y Complica- ciones [Internet]. Chguv.San.Gva.Es. 2012. Available from: http://chguv.san.gva.es/Inicio/ServiciosSalud/ServiciosHospitalarios/Documents/CirugiaGeneral/RESERVORIOS ACCESO VENOSO DE LARGA DURACIÓN ABORDAJE Y COMPLICACIONES.pdf

10. Torres Pérez JL, Gerson R, Villalobos A, Serrano A, González JM, Garmilla J, et al. Reservorio venoso subcutáneo. Reporte de 105 casos. mediagrahic Artemisa [Internet]. 2006 [cited 2017 Oct 3];51:11-7. Available from: https://books.google.es/books?hl=es \& Ir =\&id=JvAyKnw9b4C\&oi=fnd\&pg=PA22\&dq=historia + de + los + cat éteres+venosos+centrales\&ots $=0 \mathrm{~K} 1 \mathrm{AuDkqtN} \& \mathrm{sig}=$ US6RF8yXIOQRIgVWn6aPG3oFleU\#v=onepage\& $q \& f=f a l s e$

11. Kreis $\mathrm{H}$, Loehberg CR, Lux MP, Ackermann S, Lang W, Beckmann MW, et al. Patients' attitudes to totally implantable venous access port systems for gynecological or breast malignancies. Eur J Surg Oncol. 2007;33(1):39-43.

12. Toril Rubio $M$, Rodirguez Borrego $M$. Revisión sistemática de las complicaciones de los dispositivos de administración de tratamiento al paciente oncológico. Enferm Glob. 2017;16(2): 544-61.

13. Camp-Sorrell D. State of the Science of Oncology Vascular Access Devices. Semin Oncol Nurs [Internet]. 2010;26(2):80-7. Available from: http://dx.doi.org/10.1016/j.soncn.2010.02.001

14. Nagel SN, Teichgräber UKM, Kausche S, Lehmann A. Satisfaction and quality of life: a survey-based assessment in patients with a totally implantable venous port system. Eur J Cancer Care (Engl) [Internet]. 2011 Mar [cited 2017 Jan 17];21(2):197-204. Available from: http://doi.wiley.com/10.1111/j.1365-2354.2011.01275.x

15. Ullman A, Cooke M, Mitchel M, Lin F, New $\mathrm{K}$, Long $\mathrm{D}$, et al. Dressing and securement for central venous access devices (CVADs): A Cochrane systematic review. Int J Nurs Stud [Internet]. 2016 Jul 1 [cited 2017 Oct 3];59:177-96. Available from: http://www.sciencedirect.com/science/article/pii/ S0020748916300219

16. Fernández-de-Maya J, Richart-Martínez M. Variability in management of implantable ports in oncology outpatients. Eur J Oncol Nurs. 2013;17(6):835-40.

17. Dal Molin A, Guerretta L, Mazzufero F, Rasero L. The management of totally implanted venous ports in the ambulatory oncologic patient. J Vasc Access [Internet]. 2009 [cited 2017 Jan 17]; 10(1):22-6. Available from: http://www.ncbi.nlm.nih.gov/pubmed/19340795

18. Pires NN, Vasques $\mathrm{Cl}$. Nurses' knowledge regarding the handling of the totally-implanted venous access device. Texto Context - Enferm [Internet]. 2014;23(2):443-50. Available from: http:// 
w w w. s c i e lo.b r / s c i e lo.ph p ? script=sci_arttext\&pid=S0104-0707201400020044 $3 \& \operatorname{lng}=$ en\&tIng=en

19. Fernández-de-Maya J, Richart-Martínez M. Factors associated with variability in management of vascular access ports. Eur J Cancer Care (Engl). 2015;25(5):871-82.

20. Mamédio $\mathrm{C}$, Roberto $\mathrm{M}$, Nobre $\mathrm{C}$. the Pico Strategy for the Research Question. Rev latino-am Enferm. 2007;15(3):1-4.

21. Urrútia G, Bonfill X. Declaración PRISMA: una propuesta para mejorar la publicación de revisiones sistemáticas y metaanálisis. Med Clin (Barc). 2010;135(11):507-11.

22. Vandenbroucke JP, Von Elm E, Altman DG, Gøtzsche PC, Mulrow CD, Pocock SJ, et al. Mejorar la comunicación de estudios observacionales en epidemiología (STROBE): explicación y elaboración. Gac Sanit. 2009;23(2):1-28.

23. Cano Arana A, González Gil T, Cabello Lópz JB. 11 preguntas para ayudarte a entender un estudio cualitativo. Guías CASPe. 2005;3-8.

24. García de Yébenes Prous MJ, Rodríguez Salvanés F, Carmona Ortells L. Validación de cuestionarios. Reumatol Clin. 2009;5(4):171-7.

25. Tong A, Sainsbury P, Craig J. Consolidated criteria for reporting qualitative research (COREQ): A 32-item checklist for interviews and focus groups. Int J Qual Heal Care [Internet]. 2007 Sep 16 [cited 2017 Oct 22];19(6):349-57. Available from: https://academic.oup.com/intqhc/article-lookup/doi/10.1093/intqhc/mzm042

26. Ullman AJ, Cooke M, Kleidon T, Rickard CM. Road map for improvement: Point prevalence audit and survey of central venous access devices in paediatric acute care. J Paediatr Child Health. 2017;53(2):123-30.

27. Russell E, Chan RJ, Marsh N, New K. A point prevalence study of cancer nursing practices for managing intravascular devices in an Australian tertiary cancer center. Eur J Oncol Nurs [Internet]. 2014;18(3):231-5. Available from: http://dx.doi.org/ 10.1016/j.ejon.2013.11.010

28. Kapucu S, Özkaraman A, Uysal N, Bagcivan G, Şeref FÇ, Elöz A. Knowledge level on administration of chemotherapy through peripheral and central venous catheter among oncology nurses. Asia-Pacific J Oncol Nurs [Internet]. 2017 [cited 2017 Oct 4];4(1):61-8. Available from: http:// www.ncbi.nlm.nih.gov/pubmed/28217732

29. Simon A, Graf N, Furtwängler R. Results of a multicentre survey evaluating clinical practice of port and Broviac management in paediatric oncology. Klin Pädiatrie [Internet]. 2013;225(3):145-51.
Available from: http://www.ncbi.nlm.nih.gov/pub$\mathrm{med} / 23615918$

30. Özden D, Çalişkan N. Turkish nurses' level of knowledge regarding implantable port catheter care. Japan J Nurs Sci. 2012;9(1):1-8.

31. Filbet $M$, Larkin $P$, Chabloz $C$, Chirac $A$, Monsarrat L, Ruer M, et al. Barriers to venipuncture-induced pain prevention in cancer patients: $A$ qualitative study. BMC Palliat Care [Internet]. 2017 Jan 17 [cited 2017 Oct 14];16(1):1-7. Available from: http://www.ncbi.nlm.nih.gov/pubmed/ 28095834

32. Broadhurst D, Moureau N, Ullman AJ. Central venous access devices site care practices: An international survey of 34 countries. J Vasc Access. 2016;17(1):78-86.

33. WHO. Higiene de las manos [Internet]. 2015. Available from: http://www.who.int/gpsc/ 5may/tools/ES_PSP_GPSC1_Higiene-de-las-Manos_Brochure_June-2012.pdf

34. Simón Melchor A, Simón Melchor L, Naranjo Soriano G, Gil Salvador R, Solano Castrán J, Jiménez Sesma M. Importancia de la higiene de manos en el ámbito sanitario. Rev Ridec [Internet]. 2016;9(1):27-34. Available from: http://www.enfermeria21.com/revistas/ridec/pagina/normas-depublicacion/\%0Ahttp://www.enfermeria21.com/ revistas/ridec/articulo/27120/

35. Chicharro Luna E, Pertegal Vázquez F, Pérez Prieto M, Baño Ruíz L, Pérez Saura I, Pérez Bernabeu E. Clorhexidina versus povidona yodada como antiséptico de la piel. 209AD;91:1122-32. Available from: http://vapox.com/sitefiles/noticiasdocs/50/arDocumento_28_2_1.pdf

36. del Rio Carbajo L, Vidal Cortés P. Tipos de antisépticos, presentaciones y normas de uso. Med Intensiva [Internet]. 2018 [cited 2018 Dec 18]; Available from: https://www.clinicalkey.es/\#!/content/playContent/1-s2.0-S0210569118302754? returnurl=null\&referrer=null

37. Rodriguez-Barbero MP. Tipo de canalización de acceso venoso central: el PORT-A-CATH. Ene [Internet]. 2008;2(4):45-50. Available from: http://ene-enfermeria.org/ojs/index.php/ENE/article/view/168/151

38. Arroyo de Cordero G. Actualización en enfermería, una responsabilidad profesional. Rev Enferm IMSS [Internet]. 2002;10(1):3-6. Available from: http://www.medigraphic.com/pdfs/enfermeriaimss/eim-2002/eim021b.pdf

39. Arribas Martín M. Diseño y validación de cuestionarios. Matronas Profesión [Internet]. 2004;5(17):23-9. Available from: http://www.enferpro.com/documentos/validacion_cuestionarios.pdf 


\section{TABLAS Y FIGURAS}

\begin{tabular}{|c|c|c|c|c|c|c|c|c|c|c|c|c|c|c|c|c|c|c|c|c|c|c|c|}
\hline \multicolumn{24}{|l|}{ Declaración Strobe } \\
\hline \multirow[t]{2}{*}{ Autores } & \multirow{2}{*}{$\begin{array}{c}\begin{array}{c}\text { Título y } \\
\text { resumen }\end{array} \\
1\end{array}$} & \multicolumn{11}{|c|}{ Métodos } & \multicolumn{5}{|c|}{ Resultados } & \multicolumn{4}{|c|}{ Discusión } & \multirow{2}{*}{$\begin{array}{c}\begin{array}{c}\text { Otra } \\
\text { informa } \\
\text { ción }\end{array} \\
22\end{array}$} & \multirow[t]{2}{*}{ Puntos } \\
\hline & & 2 & 3 & 4 & 5 & 6 & 7 & 8 & 9 & 10 & 11 & 12 & 13 & 14 & 15 & 16 & 17 & 18 & 19 & 20 & 21 & & \\
\hline Dal Molín et al & $\mathrm{N}$ & A & A & $\mathrm{N}$ & A & A & A & $\mathrm{N}$ & N & $\mathrm{N}$ & $\mathrm{N}$ & $\mathrm{N}$ & $\mathrm{N}$ & N & A & A & $\mathrm{N}$ & A & N & A & A & $A$ & $11 / 22$ \\
\hline Özden\&Çaliskan & A & A & A & A & A & A & $\mathrm{N}$ & $N$ & A & A & A & A & $N$ & A & A & A & A & A & A & A & A & $\mathrm{N}$ & $18 / 22$ \\
\hline Simon et al & A & A & A & A & A & N & $\mathrm{N}$ & A & N & $\mathrm{N}$ & $\mathrm{N}$ & $\mathrm{N}$ & $N$ & A & A & N & A & A & A & A & A & A & $14 / 22$ \\
\hline $\begin{array}{l}\text { F.Maya\&R.Martíne } \\
z\end{array}$ & A & A & A & A & A & A & A & $N$ & A & A & A & A & A & A & A & A & A & A & A & A & A & A & $21 / 22$ \\
\hline Russell et al & A & A & A & A & A & A & A & A & A & A & $\mathrm{N}$ & A & A & A & A & N & A & A & A & A & A & A & $20 / 22$ \\
\hline Keogh et al & A & A & A & A & A & A & A & A & A & A & A & A & A & A & A & A & A & A & A & A & A & A & $22 / 22$ \\
\hline Broadhurst et al & A & A & A & A & A & A & A & $\mathrm{N}$ & $\mathrm{N}$ & A & A & A & A & A & A & A & $\mathrm{N}$ & A & A & A & $A$ & A & $19 / 22$ \\
\hline $\begin{array}{l}\text { F.Maya\&R.Martíne } \\
z\end{array}$ & A & A & A & A & A & A & A & A & N & A & A & A & A & A & A & A & A & A & A & A & $A$ & A & $21 / 22$ \\
\hline Kapucu et al & A & A & A & A & A & A & $N$ & $N$ & N & A & A & A & A & A & A & A & A & A & $\mathrm{N}$ & A & A & A & $18 / 22$ \\
\hline Ullman et al & A & A & A & A & A & A & A & A & $\mathrm{N}$ & A & $\mathrm{N}$ & A & A & A & A & A & $\mathrm{N}$ & A & A & A & A & A & 19/22 \\
\hline
\end{tabular}

Anexo I: Aplicación declaración STROBE (22) en los documentos de la revisión. (A= Afirmativo; N=Negativo.)

\begin{tabular}{|c|c|c|c|c|c|c|c|c|c|c|}
\hline \multicolumn{11}{|c|}{ CASPe estudios cualitativos } \\
\hline Autores & Objetivos & $\begin{array}{c}\text { Metodo } \\
\text { logía }\end{array}$ & Método & $\begin{array}{l}\text { Selección } \\
\text { participantes }\end{array}$ & $\begin{array}{l}\text { Recogida } \\
\text { de datos }\end{array}$ & Reflexividad & $\begin{array}{c}\text { Aspectos } \\
\text { éticos }\end{array}$ & $\begin{array}{c}\text { Análisis } \\
\text { datos }\end{array}$ & $\begin{array}{l}\text { Exposición } \\
\text { resultados }\end{array}$ & Aplicabilidad \\
\hline $\begin{array}{l}\text { N.Pyres\&I. } \\
\text { Vasques }\end{array}$ & A & A & A & A & A & - & A & A & A & A \\
\hline Filbet et al & A & A & A & A & A & - & A & A & A & A \\
\hline
\end{tabular}

Anexo II: Aplicación de CASPe en estudios cualitativos (23). (A= Afirmativo). 
VARIABILIDAD EN LA PRÁCTICA CLÍNICA DE LOS CUIDADOS DE LOS SISTEMAS DE ACCESO VenOsO

\begin{tabular}{|l|c|c|c|c|}
\hline \multicolumn{1}{|c|}{ Autoría } & Viabilidad & Fiabilidad & Validez & $\begin{array}{c}\text { Sensibilidad al } \\
\text { cambio }\end{array}$ \\
\hline Dal Molin et al & - & - & - & - \\
\hline $\begin{array}{l}\text { Ö z d e n } \\
\text { \&Çaliskan }\end{array}$ & - & - & - \\
\hline Simon et al & - & - & - \\
\hline $\begin{array}{l}\text { Fdez de Maya \& } \\
\text { Richart Minez }\end{array}$ & & - & - & - \\
\hline Russell et al & & - & & - \\
\hline Keogh et al & & - & & - \\
\hline $\begin{array}{l}\text { Broadhurst et } \\
\text { al }\end{array}$ & & & & \\
\hline Kapucu et al & & & & \\
\hline $\begin{array}{l}\text { Fdez de Maya \& } \\
\text { Richart Minez }\end{array}$ & & & & \\
\hline Ullman et al & & & & \\
\hline
\end{tabular}

Anexo III: Cumplimiento de criterios de validación de los cuestionarios.

\begin{tabular}{|l|c|}
\hline \multicolumn{1}{|c|}{ Autoría } & COREQ \\
\hline N. Pires \& I. Vasques & - \\
\hline Filbet et al & \\
\hline
\end{tabular}

Anexo IV: Cumplimiento COREQ 
